\title{
THREE COUNTEREXAMPLES CONCERNING $\omega$-CHAIN COMPLETENESS AND FIXED POINT PROPERTIES
}

\author{
by J. D. MASHBURN
}

(Received 25th April 1980)

A partially ordered set, is $\omega$-chain complete if, for every countable chain, or $\omega$-chain, in $P$, the least upper bound of $C$, denoted by sup $C$, exists. Notice that $C$ could be empty, so an $\omega$-chain complete partially ordered set has a least element, denoted by 0 .

A function $f$ mapping a partially ordered set $P$ into a partially ordered set $Q$ is chain continuous if for any nonempty chain $C$ in $P$, which has a supremum, $f\left(\sup _{P} C\right)=$ $\sup _{Q} f(C)$. It is $\omega$-chain continuous if for any nonempty countable chain $C$ in $P$, which has a supremum, $f\left(\sup _{P} C\right)=\sup _{Q} f(C)$. A partially ordered set $P$ has the least fixed point property if every order-preserving function from $P$ to itself has a least fixed point. It has the fixed point property if every order-preserving function from $P$ to itself has a fixed point. It has the least fixed point property for $\omega$-chain continuous functions if every $\omega$-chain continuous function from $P$ to itself has a least fixed point.

In 1955 A. Tarski (13, Thm. 1) and A. C. Davis (2, Thm. 2) characterised complete lattices as those lattices having the fixed point property. In 1976 G. Markowsky (6, Thm. 11) characterised the partially ordered sets having the least fixed point property as those which are chain complete. With the development by Dana Scott $(8,9)$ of lattices as models in theoretical computer science and the generalisation of these ideas by Smyth and Plotkin (12) and M. Wand (14) it has become important to know what kinds of partially ordered sets have the least fixed point property for $\omega$-chain continuous functions. In view of Markowsky's characterisation it was natural for Plotkin to ask in Pisa in 1978 if $\omega$-chain completeness follows from the least fixed point property for $\omega$-chain continuous functions. Kolodner has shown in (5) that the converse is true. The following example shows that the answer to Plotkin's question is no.

Example 1. Let $P_{1}$ be the partially ordered set defined by the diagram on the next page. Let $C=\left\{c_{n} \mid n \in \mathbb{N}\right\}, X=\left\{x_{n} \mid n \in \mathbb{N}\right\}$ and $Y=\left\{y_{n} \mid n \in \mathbb{N}\right\}$. Then $x=\sup X$ and $C$ has no supremum in $P$.

Proposition 1. Every $\omega$-chain continuous function from $P_{1}$ to itself has a least fixed point.

Proof. Let $f: P_{1} \rightarrow P_{1}$ be an $\omega$-chain continuous function with $f(0) \neq 0$. Because $P_{1}-C$ is $\omega$-chain complete; if $f(0) \in P_{1}-C$, then $f$ has a least fixed point. Assume that 


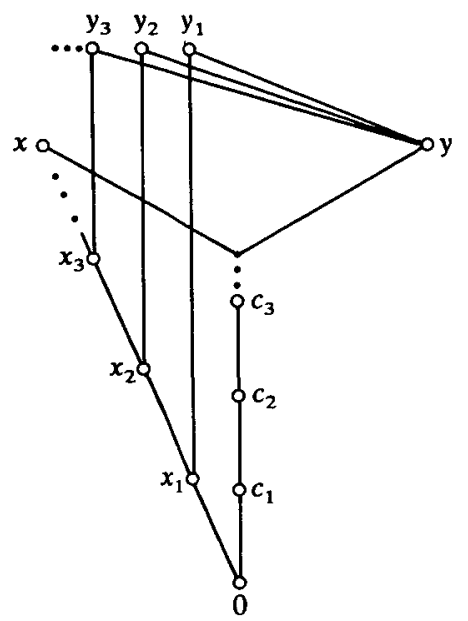

$f(0) \in C$ and that $f$ has no fixed point in $C$. If it did, it would have a least fixed point in $C$, which would be its least fixed point in $P_{1}$.

Since $f(x)=\sup f(X)$, there is an $m \in \mathbb{N}$ such that for every $n \geqq m, f\left(x_{n}\right)=f(x)$. This is because no infinite chain in the image of $f$ has a supremum.

Assume that $f(x)=x$. Then for all $n \geqq m, f\left(y_{n}\right)=x$, since $f\left(y_{n}\right) \geqq f\left(x_{n}\right)=x$. The point $y$ is below all of these, so $f(y) \leqq x$. Because we have assumed no fixed points in $C$, $f(y)=x$. Now, for all $n<m, y<y_{n}$, so $f(y) \leqq f\left(y_{n}\right)$. Thus $f\left(y_{n}\right)=x$, and $x$ is the only fixed point of $f$.

Assume that $f(x) \neq x$. The $f(y)$ is the least fixed point of $f$. This is obvious if $f(y)=y$. Assume $f(y) \neq y$. Again by the assumption that $f$ has no fixed point in $C, f(x) \geqq y$. Thus for every $n \geqq m, f\left(y_{n}\right) \geqq y$. Therefore $f(y) \in Y$. Since $y \leqq y_{n}$ for all $n \in \mathbb{N}, f(y)=f\left(y_{n}\right)$; so $f(y)$ is the only fixed point of $f$.

In (12) Smyth and Plotkin used the fact that this least fixed point will be $\sup \left\{f^{n}(0) \mid n \in \mathbb{N}\right\}$ in order to find the least fixed point of an $\omega$-chain continuous function from an $\omega$-chain complete partially ordered set to itself. However, if the condition of $\omega$-chain completeness is relaxed, this supremum may not exist, as is demonstrated by the poset $\boldsymbol{P}_{1}$ defined above. In Example 2 below this supremum will always exist even though the partially ordered set is not $\omega$-chain-complete.

The following theorem is due to R. E. Smithson (11, Thm. 1.2).

Theorem 1. Let $P$ be a partially ordered set with zero such that for every chain $C \subset P$, the set of upper bounds of $C$ is down-directed. If every order-preserving function $F: P \rightarrow P$ has a fixed point, then $P$ is chain complete.

Corollary 1. Let $P$ be a meet-semilattice. $P$ is chain-complete if and only if every order-preserving function from $P$ to itself has a fixed point.

This is not the situation for $\omega$-chain continuous functions and $\omega$-chain completeness 
as is shown by

Example 2. Let $\mathbb{N}_{\infty}=\mathbb{N} \cup\{\infty\}$ and let $Q=\left(\mathbb{N} \times \mathbb{N}_{\infty}\right) \cup\{0\}$ with the following order: for any $p, q \in Q, p \leqq q$ if and only if

(1) $p=0$ or

(2) $p=(j, k), q=(n, m)$ and either $m=\infty$ and $j \leqq n$ or $j=n$ and $k \leqq m$.

$Q$ can be represented by the diagram below. Let $X=\mathbb{N} \times\{\infty\}$ and, for every $n \in \mathbb{N}$, $X_{n}=\{n\} \times \mathbb{N}$, Now form the free meet-semilattice $\mathscr{F}\left(\mathbb{N}^{N}\right)=\left\{A \subset \mathbb{N}^{N} \mid A\right.$ is finite $\}$ ordered by containment, i.e., $A \leqq B$ if and only if $A \supset B$. Let $P=Q \cup \mathscr{F}\left(\mathbb{N}^{N}\right),(j, k) \leqq A$ if and only if for every $\left(n_{1}, n_{2}, \ldots\right) \in A, k \leqq n_{j}$.

Of course, we set $0 \leqq A$ for any $A \in \mathscr{F}\left(\mathbb{N}^{N}\right)$ to preserve transitivity. Notice that $P$ is a partially ordered set, but not a meet-semilattice since for any $(j, \infty) \in Q$ and $A \subset \mathscr{F}\left(\mathbb{N}^{N}\right)$, $(n, 1) \leqq(j, \infty)$ and $(n, 1) \leqq A$ for all $n \in \mathbb{N}$ such that $n \leqq j$, and these points $(1,1), \ldots,(j, 1)$ are not comparable. Thus we must add one more set of points.

Give $\mathbb{N} \times \mathscr{F}\left(\mathbb{N}^{N}\right)$ the usual product order and let $P_{2}=P \cup \mathbb{N} \times \mathscr{F}\left(\mathbb{N}^{N}\right)$. Relate the points of $\mathbb{N} \times \mathscr{F}\left(\mathbb{N}^{N}\right)$ to those of $P$ by the following rules: Let $(n, A) \in \mathbb{N} \times \mathscr{F}\left(\mathbb{N}^{N}\right)$

(1) for all $B \in \mathscr{F}\left(\mathbb{N}^{N}\right),(n, A) \leqq B$ if and only if $A \supset B$;

(2) for all $(m, \infty) \in Q,(n, A) \leqq(m, \infty)$ if and only if $n \leqq m$;

(3) for all $(j, k) \in Q,(j, k) \leqq(n, A)$ if and only if $(j, k) \leqq(n, \infty)$ and $(j, k) \leqq A$.

(4) $0 \leqq(n, A)$

Clearly for any $(n, \infty) \in Q$ and $A \in \wedge\left(\mathbb{N}^{N}\right),(n, \infty) \wedge A=(n, A)$. Since $Q$ and $\mathscr{F}\left(\mathbb{N}^{N}\right)$ were already meet-semilattices, $P_{2}$ is a meet-semilattice.

Notice that $X=\{(n, \infty) \mid n \in \mathbb{N}\}$ is an unbounded $\omega$-chain in $P_{2}$. In fact, it is the only incomplete $\omega$-chain in $P_{2}$. Any chain in $\mathscr{F}\left(\mathbb{N}^{N}\right)$ is finite and hence has a supremum. Also, since each of the chains $X_{n}=\{(n, m) \mid m \in \mathbb{N}\}$ where $n \in \mathbb{N}$ is complete $\left(\sup X_{n}=(n, \infty)\right)$, all of their subchains will be complete. Let $C=\left\{\left(n_{i}, A_{j}\right) \mid j \in \mathbb{N}\right\}$ be a chain in $\mathbb{N} \times \mathscr{F}\left(\mathbb{N}^{N}\right)$. If $\left\{n_{j} \mid j \in \mathbb{N}\right\}$ is finite, then $C$ must be finite. Assume that $\left\{n_{j} \mid j \in \mathbb{N}\right\}$ is infinite. Then $C$ is bounded only by elements of $\mathscr{F}\left(\mathbb{N}^{N}\right)$. Let $A=\bigcap_{j=1}^{\infty} A_{j}$. Because $A_{1} \supset A_{2} \supset \ldots$ and all the $A_{j}$ 's are finite, $A \neq \varnothing$. Let $B \in \mathscr{F}\left(\mathbb{N}^{N}\right)$ such that $B$ is an upper bound on $C$. Then for every $j \in \mathbb{N}, A_{j} \supset B$ and therefore $A \supset B$. Thus $A=\sup C$. It follows that an $\omega$-chain in $P_{2}$ is incomplete only if all but a finite number of its elements are in $X$.

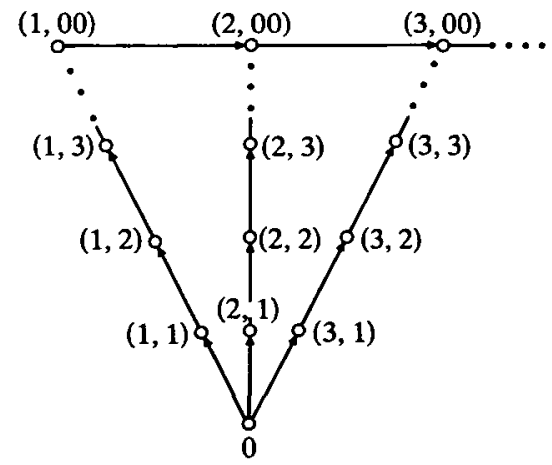


Proposition 2. Every $\omega$-chain continuous function from $P_{2}$ to itself has least fuxed point.

Proof. Let $f: P_{2} \rightarrow P_{2}$ be an $\omega$-chain continuous function with $f(0) \neq 0$. Let $F=$ $\left\{f^{n}(0) \mid n \in \mathbb{N}\right\}$. Assume that $F$ does not have a least upper bound. By the discussion above, all but a finite number of elements of $F$ are in $X$. In fact let us assume that $F \subset X$. Thus for all $n \in \mathbb{N}, X_{n} \cap f\left(P_{2}\right)=\varnothing$.

Now, since $F$ is not finite, for every $n \in \mathbb{N}$ there is an $m \in \mathbb{N}$ such that $f^{m}(0) \leqq(n, \infty)<$ $f^{m+1}(0)$. Hence $(n, \infty)<f^{m+1}(0)=f\left(f^{m}(0)\right) \leqq f((n, \infty))$. Because $f$ is $\omega$-chain continuous, there exists $\left(n_{1}, n_{2}, \ldots\right) \in \mathbb{N}^{N}$ such that for every $j \in \mathbb{N}$, and every $\left.k \geqq n_{i}, f(j, k)\right)=$ $f((j, \infty))$. In particular, for every $j \in \mathbb{N}, f\left(\left(j, n_{j}\right)\right)=f((j, \infty))$. But for every $j \in \mathbb{N},\left(j, n_{j}\right)<$ $\left\{\left(n_{1}, n_{2}, \ldots\right)\right\}$. Thus for every $j \in \mathbb{N},(j, \infty)<f((j, \infty))=f\left(\left(j, n_{j}\right)\right) \leqq f\left(\left\{\left(n_{1}, n_{2}, \ldots\right)\right\}\right)$. This is impossible since $X$ has no upper bound.

If $F \notin X$, then $f(0) \notin X$. If $f(0) \in X_{n}$ for some $n$, then $X_{n} \cap f\left(P_{2}\right) \neq \varnothing$, but this is not true for any other $X_{m}$. If $f(0) \notin X_{n}$ for any $n \in \mathbb{N}$, then for all $n \in \mathbb{N}, X_{n} \cap f\left(P_{2}\right)=\varnothing$. In either case the argument used above shows that $F$ must have a supremum, which is the least fixed point of $f$.

A retract from a partially ordered set to some nonempty subset is an $\omega$-chain continuous function which leaves the points of the subset fixed. The subset is then called a retraction of the partially ordered set. One obvious necessary condition for a partially ordered set $P$ to have the least fixed point property for $\omega$-chain continuous function is the following.

(R) If $C$ is a nonempty $\omega$-chain in $P, U$ is a set of upper bounds of $C$, and there is a retraction from $P$ to $P$ to $C \cup U$, then $U$ has a least element.

In particular no unbounded $\omega$-chain is a retraction of $P$. Both $P_{1}$ and $P_{2}$ have this property.

Markowsky used a similar property for order preserving functions in his characterisation. But this condition is not sufficient. To see this an equivalent topological condition is first needed.

Definition. Let $P$ be a partially ordered set. Define $\kappa(P)$ to be the collection of all $U \subset P$ such that if $D$ is a nonempty countable directed subset of $P$ and $\sup D \in U$, then $U \cap D \neq \varnothing$.

Notice that any such directed set $D$ has a well ordered cofinal $\omega$-chain and so the directed set in the definition could be replaced by an $\omega$-chain. Also, if $U \in \kappa(P)$ and $C$ is a non-empty $\omega$-chain in $P$ with a supremum in $P$, then there is some $c \in C$ so that $\{d \in C \mid c \leqq d\} \subset U$. If not, then $C-U$ would be cofinal in $C$ and hence be a nonempty $\omega$-chain with a supremum in $U$ which did not intersect $U$. From these observations it is easy to show that $\kappa(P)$ is a topology. In fact it is the finest topology with the property that if $C$ is a nonempty $\omega$-chain in $P$ and $p \in P$ then $p=\sup C$ if and only if every open neighborhood of $p$ intersects $C$. By restricting $\kappa(P)$ to those sets which are also increasing, a topology very much like the Scott-topology (see (8)) is obtained.

Lemma 1. A subset $X$ of $P$ is closed relative to $\kappa(P)$ if and only if $X$ is closed with respect to supremums of nonempty $\omega$-chains. 
The proof is easy. It follows that for any $p \in P, \downarrow p=\{q \in P \mid q \leqq p\}$ is both open and closed in $\kappa(P)$ and hence that $\kappa(P)$ is Hausdorff. In fact the order of $P$ is closed in the product $P \times P$ relative to $\kappa(P)$.

Proposition 3. Let $P$ and $Q$ be partially ordered sets. An order preserving function $f: P \rightarrow Q$ is $\omega$-chain continuous if and only if $f$ is continuous with respect to $\kappa(P)$ and $\kappa(Q)$.

Proof. Assume that $f$ is $\omega$-chain-continuous and let $U \in \kappa(Q)$. Let $C \subset P$ be a nonempty $\omega$-chain with $\sup _{P} C \in f^{-1}(U)$. Then $\sup _{Q} f(c)=f\left(\sup _{p} C\right) \in U$ so that $f(C) \cap U \neq \varnothing$. Thus $C \cap f^{-1}(U)=\varnothing$ and $f^{-1}(U) \in \kappa(P)$

Now assume that $f$ is topologically continuous. Let $C \subset P$ be a nonempty $\omega$-chain with a supremum in $P$ and let $U \in \kappa(Q)$ such that $f\left(\sup _{P} C\right) \in U$. Then $\sup _{P} C \in$ $f^{-1}(U) \in \kappa(P)$ and $C \cap f^{-1}(U) \neq \varnothing$. Therefore $f(C) \cap U \neq \varnothing$ and so $f\left(\sup _{P} C\right)=$ $\sup _{Q} f(C)$.

Corollary 2. Let $P$ be a partially order set. An unbounded $\omega$-chain $C=\left\{c_{n} \mid n \in \mathbb{N}\right\}$ is a retraction of $P$ if and only if there is a collection of disjoint $\kappa$-open and closed subsets of $P$ such that $P=\bigcup_{n \in N} D_{n}$, for each $n, c_{n} \in D_{n}$, and if $p \in D_{n}$ and $q \leqq p$, then $q \in D_{m}$ for some $m \leqq n$.

Proof. If $C$ is a retraction of $P$ and $f: P \rightarrow C$ is a retract, take $D_{n}=f^{-1}\left(C_{n}\right)$. If such a collection of open and closed sets exists, the function $f: P \rightarrow C$ defined by $f(p)=C_{n}$ if and only if $p \in D_{n}$ is a retract. It preserves the sups of nonempty countable chain since, if $A$ is a nonempty $\omega$-chain with a supremum then sup $A \in D_{n}$ for some $n$ and thus, because $D_{n}$ is open, there exists $b \in A$ so that $\{a \in A \mid b \leqq a\} \subset D_{n}$.

Example 3. Let $P_{3}$ be the subset of $\mathbb{N}_{\infty}^{N}$ consisting of all elements $\bar{x}=\left(x_{1}, x_{2}, \ldots\right)$ such that $x_{n}=\infty$ for only a finite number of $n$ 's and $x_{n+1}=\infty$ implies $x_{n}=\infty$. Order $P_{3}$ with the usual product order. Then $P_{3}$ is a lattice and every nonempty subset of $P_{3}$ has an infimum. Thus every bounded $\omega$-chain in $P_{3}$ has a supremum.

Proposition 4. $P_{3}$ satisfies condition $R$.

Proof. If $C$ is a nonempty bounded $\omega$-chain, $U$ is a set of upper bounds of $C$ and $f: P \rightarrow C \cup U$ is a retract, then $\sup C \in U$, since $f(\sup C)=\sup f(C)=\sup C$. Therefore $U$ has a least element. To show that no unbounded $\omega$-chain is a retraction of $P$, only one chain need be considered because an $\omega$-chain continuous function can always be found between one $\omega$-chain and another.

For every $n \in \mathbb{N}$, let $\bar{c}_{n}$ be the element of $P_{3}$ having $\infty$ as its first $n$ components and 1 everywhere else. Then $C=\left\{\bar{c}_{n} \mid n \in \mathbb{N}\right\}$ is an unbounded $\omega$-chain. Let $D=\left\{D_{n} \mid n \in \mathbb{N}\right\}$ be a collection of disjoint $\mu$-open and closed subsets of $P_{3}$ such that for each $n, \bar{c}_{n} \in D_{n}$ and if $p \in D_{n}$ and $q \leqq p$, then $q \in D_{m}$ for some $m \leqq n$. For every $n$, let $E_{n}=\bigcup_{m \leq n} D_{m}$.

Set $x_{1}=1$. Assume that $x_{k}$ has been defined for every $k \leqq n$. Let $X_{n}=$ $\left\{x \in \mathbb{N} \mid\left(x_{1}, \ldots, x_{n}, x, 1,1, \ldots\right) \in E_{n}\right\}$. If $X_{n}=\varnothing$, set $x_{n+1}=1$. If not, then it must be finite since $E_{n}$ is closed and $\bar{c}_{n+1} \notin E_{n}$. In this case set $x_{n+1}=\left(\max X_{n}\right)+1$. 
Each $x_{n}$ defined by the above process is a positive integer so $\bar{x}=\left(x_{1}, x_{2} \ldots\right) \in P_{3}$. But $\bar{x}$ is not contained in any $D_{n}$. If $\bar{x} \in D_{n}$ for some $n$, then $\bar{y}=\left(x_{1}, \ldots, x_{n+1}, 1,1, \ldots\right) \in D_{m}$ for some $m \leqq n$ since $\bar{y} \leqq \bar{x}$. Thus $\bar{y} \in E_{m}$ which contradicts the definition of $x_{n+1}$. Therefore by Corollary 2 , no unbounded $\omega$-chain is a retraction of $P_{3}$.

Nevertheless, $\boldsymbol{P}_{3}$ does not have the least fixed point property for $\boldsymbol{\omega}$-chain continuous functions. The function $f: P_{3} \rightarrow P_{3}$ defined by $f\left(x_{1}, x_{2}, \ldots\right)=\left(x_{1}+1, x_{2}+1, \ldots\right)$ is an $\omega$-chain continuous function with no fixed points.

\section{REFERENCES}

(1) S. Abian and A. B. Brown, A theorem on partially ordered sets with applications to fixed point theorems, Canadian J. Math. 13 (1961), 78-82.

(2) A. C. Davis, A characterization of complete lattices, Pacific J. Math. 5 (1955), 311-319.

(3) R. DemarR, Common fixed points for isotone mappings, Colloq. Math. 13 (1964), 45-48.

(4) HoFt and HoFt, Some fixed point theorems for partially ordered sets, Canadian J. Math. 28 (1976), 992-997.

(5) I. I. KOLODNER, On completeness of partially ordered sets and fixpoint theorems for isotone mappings, Amer. Math. Monthly 75 (1968), 48-49.

(6) G. Markowsky, Chain-complete posets and directed sets with applications, Algebra Univ. 6 (1976), 53-68.

(7) A. Pelczar, On the invariant points of a transformation, Ann. Pol. Math. 11 (1961), 199-202.

(8) Dana Scotr, Continuous Lattices, Proc. 1971 Dalhousie conference (Lecture Notes in Math. No. 274, Springer-Verlag, New York, 1972).

(9) Dana Scort, Data types as lattices, SIAM J. Computing 5 (1976), 522-587.

(10) R. E. Smrthson, Fixed points of order preserving multifunctions, Proc. Amer. Math. Soc. 28 (1971), 304-310.

(11) R. E. Smrthson, Fixed points on partially ordered sets, Pacific J. Math. 45 (1973), 363-367.

(12) M. B. Smyth and G. D. Plotkin, The Category-Theoretic Solution of Recursive Domain Equations (D.A.I. Research Report No. 60, University of Edinburgh, 1978).

(13) A. TARskr, A lattice-theoretical fixpoint theorem and its applications, Pacific J. Math. 5 (1955), 285-309.

(14) M. WAND, Fixed-point constructions in order-enriched categories, Theor. Comput. Sci. 8 (1979) 13-30.

(15) L. E. WARD, JR., Completeness in semilattices, Canadian J. Math. 9 (1957), 578-582.

(16) E. S. WoLk, Dedekind completeness and a fix-point theorem, Canadian J. Math. 9 (1956), 400-405.

(17) J. S. W. WonG, Common fixed points of commuting monotone mappings, Canadian J. Math. 19 (1966), 617-620.

UNIVERSITY OF CALIFORNIA

RIVERSDE, CA 92521 